Enfermagem Brasil 2018;17(6):576-84

https://doi.org/10.33233/eb.v17i6.1250

\title{
ARTIGO ORIGINAL \\ Caracterização do perfil de doadoras do banco de leite humano da maternidade escola de Salvador/BA
}

Yasmin Costa Santos*, Jadna Dourado Gaspar*, Géssica Brito Duarte*, Flavia Pimentel Miranda, M.Sc. **

*Enfermeira graduada pela Universidade Salvador UNIFACS/ Laureate International Universities, Salvador/BA, ${ }^{\star \star}$ Enfermeira, Professora colaboradora I da Escola de Enfermagem da Universidade Salvador UNIFACS, Laureate International Universities, Escola Bahiana de Medicina e Saúde Pública (EBMSP), Salvador/BA

Recebido 18 de outubro de 2017; aceito em 31 de maio de 2018.

Endereço para correspondência: Flavia Pimentel Miranda, Av. Luís Viana, 3146, Imbuí, 41720-200 Salvador BA, E-mail: flaviabrim@hotmail.com; Yasmin Costa Santos: yasmin.c_s@hotmail.com; Jadna Dourado Gaspar: jadnadouradogaspar@hotmail.com; Gessica Brito Duarte: gessica_tec@hotmail.com

\section{Resumo}

Objetivo: Caracterizar o perfil sociodemográfico e a história obstétrica atual das mães doadoras de leite humano de uma maternidade escola de Salvador/BA. Métodos: Estudo transversal, exploratório, descritivo com abordagem quantitativa, realizado no banco de leite humano de uma maternidade de Salvador/BA no ano de 2015. Para a coleta de dados foi utilizada uma entrevista estruturada composta por questões objetivas e subjetivas, os dados foram analisados a partir do software SPSS versão 14.0 for Windows e apresentados descritivamente sob a forma de tabelas. Resultados: As mulheres na sua maioria eram casadas multíparas, tiveram acompanhamento de pré-natal, parto normal e mantiveram o aleitamento exclusivo, eram assalariadas e possuíam bom nível de escolaridade. Conclusão: Conhecer o perfil dessas doadoras permite o adequado enfoque das ações de captação e divulgação dos Bancos de Leite Humano e favorece outras pesquisas do mesmo aspecto, com outras metodologias para elaboração de estratégias para captação de doadoras.

Palavras-chave: alimentação, aleitamento materno, bancos de leite.

\section{Abstract \\ Characterization of donor profile of human milk bank of the maternity school of Salvador/BA}

Objective: To characterize the demographic profile and the current obstetric history of human milk from donor mothers of a maternity school of Salvador, Bahia. Methods: Transversal study, exploratory, descriptive quantitative approach in human milk bank of a maternity school of Salvador/BA in the year 2015. For the collection of data we used a structured interview which consists of objective and subjective questions. Data were analyzed using the SPSS version 14.0 software for Windows and presented descriptively in the form of tables. Results: The majority of women was married, multiparous, prenatal care monitoring, natural childbirth and maintained the exclusive breastfeeding, was salaried and has good level of schooling. Conclusion: To know the profile of these donors allows the appropriate focus of collection and dissemination of human milk banks and promotes further research of the same aspect, with other methodologies for the elaboration of strategies to attract donors.

Key-words: nutrition, breastfeeding, milk banks.

\section{Resumen}

Caracterización del perfil donante del banco de leche humano de la maternidad escuela de Salvador/BA

Objetivo: Caracterizar el perfil sociodemográfico y la historia obstétrica actual de las madres donadoras de leche humano de una maternidad escuela de Salvador/BA. Métodos: Estudio transversal, exploratorio, descriptivo con abordaje cuantitativo, realizado en el banco de leche 
humano de una maternidad de Salvador/BA en el año de 2015. Para la colecta de datos fue utilizada una entrevista estructurada compuesta por cuestiones objetivas y subjetivas. Los datos fueron analizados a partir del software SPSS versión 14.0 para Windows y presentados descriptivamente en la forma de tablas. Resultados: Las mujeres en su mayoría eran casadas, multíparas, tuvieron acompañamiento prenatal, parto normal y mantuvieron la lactancia materna exclusiva, eran asalariadas y poseían alta escolaridad. Conclusión: Conocer el perfil de esas donadoras permite el adecuado enfoque de las acciones de captación y divulgación de los bancos de leche humano además de favorecer otras investigaciones del mismo aspecto, con otras metodologías para elaboración de estrategias para la captación de donadoras.

Palabras-clave: alimentación, lactancia materna, bancos de leche.

Introdução

$\mathrm{Na}$ perspectiva da prevenção e promoção da saúde do recém-nascido $(\mathrm{RN})$ e do lactente, o aleitamento materno (AM) é considerado uma estratégia fundamental para redução da morbimortalidade infantil. Consiste em uma oferta nutricional na quantidade e qualidade necessária para o desenvolvimento mental e crescimento físico do indivíduo. Entre os seus componentes estão: proteínas, ácidos graxos, enzimas e fatores de crescimento que protegem contra infecções, alergias e distúrbios gastrointestinais [1,2].

A soma das propriedades biológicas, fatores psicoafetivos e o baixo custo tornam o leite humano a escolha perfeita para alimentação do $\mathrm{RN}$, além dos benefícios gerados à mãe como: regressão uterina no pós-parto e menor prevalência de câncer de mama e ovário. Desta forma, os benefícios do aleitamento materno são proporcionados ao binômio mãe e lactente [3].

O leite humano da própria mãe é a escolha inicial de alimentação do recém-nascido principalmente dos prematuros. Entretanto, existem situações em que o leite materno não pode ser ofertado ao neonato, a exemplo de genitoras portadoras do Human Immunodeficiency Virus (HIV) [4], doenças transmissíveis como a varicela, ou ainda prematuros incapazes de sugar ao seio materno. Assim, os bancos de leite humano (BLH) se fazem como uma estratégia eficaz de promoção, proteção e apoio ao aleitamento materno $[5,6]$.

O BLH, de acordo com RDC/ANVISA n 171 de 2006, é um centro especializado sem fins lucrativos, vinculado obrigatoriamente a um hospital materno ou infantil, responsável pela promoção do incentivo ao aleitamento materno e execução das atividades de coleta, processamento e controle de qualidade de leite materno humano $(\mathrm{LMH})$, com vistas à posterior distribuição para crianças que dele necessitam para sua sobrevivência [7].

Os bancos de leite humano permitem também a construção de uma rede de possibilidades relacionadas à nucleação familiar e social, favorecendo a aquisição do leite humano pelas mães impossibilitadas de amamentar, contribuindo significativamente para a melhor interação entre elas e suas crianças [6].

Nos últimos anos as políticas públicas de saúde destinadas ao incentivo do aleitamento materno e ao fortalecimento dos BLH adquiriram destaque e expansão no cenário nacional e internacional [6], a exemplo do programa de cooperação Ibero-Americana, orientado pelo intercâmbio de conhecimento e tecnologia do aleitamento materno e bancos de leite humano, para que atinja um dos objetivos do milênio da Organização Mundial de Saúde (OMS), representado por acabar com as mortes evitáveis de recém-nascidos e crianças menores de 5 anos, para pelo menos 12 por 1.000 nascidos vivos até 2030 [8].

A rede de BLH do Brasil é uma das mais complexas e eficientes em todo mundo. Os recentes e bons resultados apresentados e as características empregadas foram fortes motivadores para o processo de exportação do modelo de BLH para diversos países, em destaque aos em desenvolvimento. Sendo isto, nada mais que uma tentativa conjunta de desenvolvimento socioeconômico de maioria populacional marginalizada [9].

No entanto os BLH muitas vezes estão com o estoque de leite humano muito baixo, não conseguindo suprir as necessidades da instituição. Na cidade de Salvador, um dos principais BLH do estado, pertencente a uma maternidade escola, enfrentou grande crise em meados $2010 \mathrm{com}$ a redução de doadoras e consequentemente o baixo estoque para atender a demanda interna [10], implicando dessa forma na diminuição da oferta do leite humano (LH) às crianças internadas.

Diante destas considerações, evidenciou-se a necessidade de conhecer o perfil de doadoras de LH, para que posteriormente possam ser elaboradas estratégias de captação dessas doadoras, e um consequente aumento do estoque de leite humano, contribuindo para redução da morbimortalidade infantil. 
Assim, objetivamos responder ao seguinte questionamento: Qual o perfil sociodemográfico e a história obstétrica das mulheres doadoras do Banco de Leite Humano de uma Maternidade Escola de Salvador/BA?

\section{Material e métodos}

Trata-se de um estudo transversal, exploratório, descritivo com abordagem quantitativa, realizado no Banco de Leite Humano da referida maternidade em Salvador/BA.

Para tanto foi construída uma entrevista estruturada composta por questões objetivas e subjetivas, contemplando às seguintes questões: dados de identificação, estado civil, escolaridade, ocupação, renda familiar, além de perguntas sobre morbidades, gestações anteriores (número de gestações, de partos e de abortos), acompanhamento no pré-natal, uso de medicamentos e contraceptivos, história da gestação atual (alterações mamárias, consulta e exames de pré-natal e tipo de parto) e saúde do concepto (peso ao nascer, semanas de gestação, tipo de alimentação e se receberam orientações durante o pré-natal).

Como critérios de inclusão para o estudo, consideraram-se mulheres que estavam cadastradas e doando LH no respectivo banco de leite, mesmo que não estivessem parido neste; e a necessidade de doação no período estipulado para coleta de dados e a assinatura o Termo de consentimento livre e esclarecido (TCLE). Os critérios de exclusão foram: pacientes com deficiência auditiva, impossibilitadas de responderem a entrevista ou que não assinaram o TCLE.

A amostra foi constituída por 31 nutrizes doadoras, que aceitaram participar da pesquisa e identificadas pelo BLH no período delimitado para coleta de dados, de 12 de julho a 20 de setembro de 2015. Para captação das doadoras internas, realizou-se um convite individual no alojamento conjunto, momento em que se esclareceu a pesquisa e posterior assinatura do TCLE. Posteriormente, a participante era entrevistada em local reservado, garantindo a privacidade e confidencialidade das informações. Para o grupo de doadoras externas, o convite para participação da pesquisa foi feito via contato telefônico, a partir da busca dessas doadoras no cadastro do próprio BLH. Foram esclarecidos os aspectos relacionados à pesquisa, e após o aceite encaminhávamos o TCLE para a residência da doadora através do corpo de bombeiro, que atualmente realiza a coleta do leite humano doado para o Instituto. Posteriormente ao recebimento do TCLE, devidamente assinado pela doadora, realizávamos um novo contato telefônico para realização da entrevista.

Os dados foram analisados a partir do software Statistical Package for Social Sciences (SPSS), versão 14.0 para Windows. A normalidade das variáveis foi verificada através da estatística descritiva e do teste Shapiro-Wilk. Os resultados foram apresentados por meio de tabelas, as variáveis categóricas expressas em valores absolutos e percentuais - $n$ (\%). As variáveis contínuas com distribuição normal foram expressas em média e desvio padrão ( $\pm D P)$, e aquelas com distribuição assimétrica, em mediana e intervalo interquartil (IQ). Para comparação entre grupos foi utilizado o teste Mann Whitney para a variável renda, o qual apresentou uma distribuição não paramétrica. As comparações das variáveis categóricas não foram possíveis de serem realizadas através do teste qui-quadrado, devido ao número amostral não ser representativo. O nível de significância adotado foi $p<0,05$.

A pesquisa foi desenvolvida dentro dos padrões éticos. Para tanto, seguimos as normas e diretrizes regulamentadoras de pesquisas com seres humanos, estabelecidas pela Resolução no 466, de 12 de dezembro de 2012 do Conselho Nacional de Saúde. O projeto foi aprovado pelo Comitê de Ética em Pesquisa (CEP) da Universidade Salvador (UNIFACS) sob n CAAE 44403215.9.0000.5033.

\section{Resultados}

A amostra foi composta por 31 doadoras, 16 eram doadoras internas (DI) e 15 eram doadoras externas (DE). Na tabela I, apresentam-se as características sociodemográficas e as comparações entre os grupos de doadoras de leite humano.

A média de idade do grupo das doadoras internas foi de 23,9 anos $( \pm 6,4 \%)$ e a média das doadoras externas foi 31,4 anos $( \pm 5,9 \%)$, com um $p=0,002$. Quanto ao estado civil em ambos os grupos de doadoras, a maior frequência foi de mulheres casadas. A situação funcional em sua maioria foi de mulheres assalariadas, com diferença significativa na renda familiar entre as doadoras ( $R \$ 6.000,00$ para DE e $R \$ 2.000$ DI, $p<0,01)$. A variável 
escolaridade apresentou uma maior frequência de ensino superior completo nas DE e as DI com o grau de escolaridade do $2^{\circ}$ grau completo conforme demonstrado na tabela abaixo.

Tabela I - Distribuição das características sociodemográficas das doadoras de leite humano de uma Maternidade Escola de Salvador/BA, 2015.

\begin{tabular}{llll}
\hline Variáveis & $\mathbf{n}(\%)$ & DI & DE \\
\hline Idade & 31 & $23,9(6.4 \%)$ & $31,4(5,9 \%)$ \\
Estado civil & & & \\
Casada & $18(58,1 \%)$ & $8(50 \%)$ & $10(66,7 \%)$ \\
Outra & $3(9,6 \%)$ & $2(12,5 \%)$ & $1(6,7 \%)$ \\
Escolaridade & & & \\
10Grau incompleto & $2(6,5 \%)$ & $1(6,3 \%)$ & $1(6,7 \%)$ \\
10Grau completo & $3(9,7 \%)$ & $3(18,8 \%)$ & 0 \\
20Grau incompleto & $3(9,7 \%)$ & $1(6,3 \%)$ & $2(13,3 \%)$ \\
20Grau completo & $12(38,7 \%)$ & $10(62,5 \%)$ & $2(13,3 \%)$ \\
Superior incompleto & $4(12,9 \%)$ & $1(6,3 \%)$ & $3(20 \%)$ \\
Situação funcional & & & \\
Estudante & $5(16,1 \%)$ & $3(18,8 \%)$ & $2(13,3 \%)$ \\
Desempregada & $7(22,6 \%)$ & $5(31,3 \%)$ & $2(13,3 \%)$ \\
Assalariada & $14(45,2 \%)$ & $5(31,3 \%)$ & $9(60 \%)$ \\
Autônoma & $5(16,1 \%)$ & $3(18,8 \%)$ & $2(13,3 \%)$ \\
Renda familiar & & & \\
Renda em R $\$$ M (IQ) & & & \\
Residem na casa m $(\mathrm{DP})$ & $2000(845-5000)$ & $2000(780-2000)$ & $6000(2000-10000)$ \\
\hline
\end{tabular}

$\mathrm{DI}=$ Doadora Interna; $\mathrm{DE}=$ Doadora Externa; $\mathrm{n}=$ número de participantes; $\mathrm{DP}=$ Desvio padrão; ${ }^{*} \mathrm{p}<0,05$ no teste Mann-Whitney; $I Q$ = Intervalo interquartil, $M=$ mediana; $m$ = média.

A maioria das doadoras era primíparas, e as $D E$ apresentaram uma maior frequência de realização de aborto, parto, filho e gestação quando comparado com as $\mathrm{DI}$, conforme demonstrado na tabela II. Há uma maior frequência de hipertensão arterial sistêmica (HAS), representando 9,7\% (n=3), seguida por tuberculose 6,5\% (n=2), em DI. Quando questionadas sobre o uso de bebida alcoólica, somente $6,5 \%(n=2)$ DI referiram fazer uso, ambos os grupos de doadoras não apresentam histórico de fumo.

Tabela II - História obstétrica e clínica, uso de medicações e hábitos de vida das doadoras de leite humano de uma Maternidade Escola de Salvador/BA, 2015.

\begin{tabular}{llll}
\hline Variáveis & $\mathbf{n}(\%)$ & $\mathbf{D I}$ & $\mathbf{D E}$ \\
\hline $\begin{array}{l}\text { Antecedentes } \\
\text { Obstétricos (m } \pm \text { DP) }\end{array}$ & & & \\
Número de gestação & $1,8 \pm 1$ & $1,5 \pm 0,5$ & $2,2 \pm 1,3$ \\
Número de Partos & $1,5 \pm 0,5$ & $1,3 \pm 0,4$ & $1,7 \pm 0,5$ \\
Número de Filhos & $1,5 \pm 0,5$ & $1,2 \pm 0,4$ & $1,7 \pm 0,5$ \\
Abortamento $\mathbf{n}(\%)$ & $8(25,8 \%)$ & $3(18,8 \%)$ & $5(33,3 \%)$ \\
História clínica $\mathbf{n}(\%)$ & & & \\
Hepatite & $1(3,3 \%)$ & $1(6,3 \%)$ & $0 \%$ \\
Chagas & $0 \%$ & $0 \%$ & $0 \%$ \\
HAS & $3(9,7 \%)$ & $3(18,8 \%)$ & $0 \%$ \\
Tuberculose & $2(6,5 \%)$ & $2(12,5 \%)$ & $0 \%$ \\
Doença Cardíaca & $1(3,2 \%)$ & $1(6,3 \%)$ & $0 \%$ \\
Transfusão de sangue & $1(3,2 \%)$ & $0 \%$ & $1(6,7 \%)$ \\
Hábitos de vida $\mathbf{n}(\%)$ & & & \\
Fuma & $0 \%$ & $0 \%$ & $0 \%$ \\
Uso de bebida alcoólica & $2(6,5 \%)$ & $2(12,5 \%)$ & $0 \%$ \\
\hline
\end{tabular}

$\mathrm{DI}=$ Doadora Interna; $\mathrm{DE}=$ Doadora Externa; $\mathrm{n}$ = número de participantes; $\mathrm{DP}=$ Desvio padrão; $\mathrm{m}$ = média.

Em relação às mamas, $62,5 \%$ das $\mathrm{DI}$ referiram que as mamas estavam em condições normais, entretanto $(46,7 \%)$ das DE relataram mamas ingurgitadas. Em relação ao tipo de parto, $58,1 \%(n=18)$ de doadoras referiram parto normal e $93,5 \%(n=29)$ realizaram pré-natal. 
Tabela III - Situação obstétrica atual das doadoras de leite humano de uma Maternidade Escola de Salvador/BA, 2015.

\begin{tabular}{llll}
\hline Variáveis & $\mathbf{n}(\%)$ & DI & DE \\
\hline Mamas & & & \\
Normais & & $10(62,5 \%)$ & $7(46,7 \%)$ \\
Ingurgitadas & $17(54,8 \%)$ & $6(37,5 \%)$ & $7(46,7 \%)$ \\
$\begin{array}{l}\text { Tipo de parto n(\%) } \\
\text { Normal }\end{array}$ & $13(41,9 \%)$ & $10(62,5 \%)$ & $8(53,3 \%)$ \\
Fez pré-natal? & $18(58,1 \%)$ & $14(87,5 \%)$ & $15(100 \%)$ \\
Sim & $29(93,5 \%)$ & $2(12,5 \%)$ & $0(\%)$ \\
Não & $2(6,5 \%)$ & $15(93,8 \%)$ & $15(100 \%)$ \\
Exames sorológicos & & $1(6,2 \%)$ & $0(\%)$ \\
Sim & $30(96,8 \%)$ & $1(3,2 \%)$ & \\
Não & & & \\
\hline
\end{tabular}

$\mathrm{DI}=$ doadora interna; $\mathrm{DE}=$ doadora externa; $\mathrm{n}=$ número de participantes.

Ao serem questionadas sobre a saúde do neonato e condições do aleitamento materno, $77,4 \%(n=24)$ referiram o nascimento dos filhos com peso adequado e $64,5 \%(n=20)$ com idade gestacional adequada, conforme tabela IV.

Observa-se diferença de frequência do tipo de aleitamento entre as doadoras, no qual, as DI possuem predominantemente o aleitamento misto (50\%), enquanto as DE em sua maioria possuem o aleitamento exclusivo $(86,7 \%)$. Em relação à última variável, $65 \%$ das doadoras afirmam ter recebido orientações dos profissionais de saúde durante o pré-natal e 0 pós-parto, com destaque para os benefícios do aleitamento exclusivo até os seis meses de idade, os malefícios do uso de chupetas, chuquinhas, mamadeiras e doação de LH.

Tabela IV- Análise descritiva da saúde do lactente e o perfil de amamentação das doadoras de leite humano de uma Maternidade Escola de Salvador/BA, 2015.

\begin{tabular}{|c|c|c|c|}
\hline Variáveis & n (\%) & DI & $\mathrm{DE}$ \\
\hline \multicolumn{4}{|l|}{ Peso ao nascer } \\
\hline Adequado (entre os percentis 10 e 90 ) & $24(77,4 \%)$ & $12(75 \%)$ & $12(80 \%)$ \\
\hline Baixo peso (abaixo do percentil 10) & $6(19,4 \%)$ & $4(25 \%)$ & $2(13,3 \%)$ \\
\hline $\begin{array}{l}\text { Acima do peso (acima do percentil } 90 \text { ) } \\
\text { Tempo de gestação } n(\%)\end{array}$ & $1(3,2 \%)$ & $0 \%$ & $1(6,7 \%)$ \\
\hline Termo (37 a 41 semanas e 6 dias) & $20(64,5 \%)$ & $9(56,3 \%)$ & $11(73,7 \%)$ \\
\hline $\begin{array}{l}\text { Pré-termo ( } \leq 36 \text { semanas e } 6 \text { dias) } \\
\text { Aleitamento do bebê }(\%)\end{array}$ & $11(35,5 \%)$ & $7(43,8 \%)$ & $4(26,7 \%)$ \\
\hline Misto & $9(29 \%)$ & $8(50 \%)$ & $1(6,7 \%)$ \\
\hline Exclusivo & $20(64,5 \%)$ & $7(43,8 \%)$ & $13(86,7 \%)$ \\
\hline Predominante materno & $2(6,5 \%)$ & $1(6,3 \%)$ & $1(6,7 \%)$ \\
\hline $\begin{array}{l}\text { Orientação sobre aleitamento materno } \\
\text { no pré-natal n (\%) }\end{array}$ & $20(64,5 \%)$ & $11(68,8 \%)$ & $9(50 \%)$ \\
\hline
\end{tabular}

Discussão

$\mathrm{Na}$ tabela I, ao analisar a variável idade, percebe-se que as mulheres possuíam em média 31 anos, sendo representado por mulheres adultas, em idade reprodutiva, resultado que corrobora outros estudos [7,11-13]. Essa faixa etária pode também representar uma maior conscientização sobre a importância do aleitamento materno e consequente doação de LH.

Tanto na condição de DI como DE, houve uma maior frequência de mulheres casadas, resultado que corrobora outros estudos [14]. Acredita-se que o apoio social oferecido pelo parceiro pode influenciar na prática do aleitamento materno. Segundo Lima et al. [15], o apoio paterno é um importante aliado na prática da amamentação e no processo de doação de LH. Entretanto em um estudo qualitativo que objetivou revelar os sentimentos vivenciados pelo pai, durante o processo de amamentação, evidenciou-se que os pais que receberam orientações da equipe tiveram melhor desempenho junto à mãe no processo de amamentação, apoiando, participando, incentivando e reconhecendo a importância do leite materno para seu filho [16]. 
Quando questionadas sobre a escolaridade, a maioria das mulheres referiu ter o $2^{\circ}$ grau e/ou ensino superior completo. Um estudo que objetivou descrever os fatores associados à prática do aleitamento materno em nutrizes de um hospital público do Distrito Federal mostrou que mulheres com maior nível de escolaridade amamentam por mais tempo [17].

Outro estudo realizado encontrou como uma das variáveis preditoras do aleitamento materno exclusivo por pelo menos 6 meses, a variável escolaridade (OR 2,1; IC95\% 1,2 - 3,6) [18]. Dessa forma, o grau de instrução da doadora pode influenciar positivamente na decisão e na adesão à prática de doação de leite, na medida em que interfere na compreensão das orientações recebidas sobre aleitamento materno.

Para ambas as categorias destacou-se como situação funcional a condição de assalariada, provavelmente representada pela inserção da mulher no mercado de trabalho. Entretanto a amamentação, segundo Nakano \& Mamede [19], é uma prática valorizada e cobrada socialmente, impondo à mulher a responsabilidade na determinação das condições de bem-estar físico e emocional do filho.

O Ministério da Saúde, em parceria com a Sociedade Brasileira de Pediatria lançou uma campanha a fim de estimular as empresas privadas a adotarem a licença maternidade de seis meses, a garantia da creche no ambiente do trabalho ou próximo dele e a implantação das salas de aleitamento materno, ambiente criado no próprio trabalho, destinados às mulheres que retornam da licença maternidade. A intenção é que elas possam durante o horário de trabalho, com privacidade e segurança, retirar o leite, armazená-lo em local adequado e depois levá-lo para casa, aumentando o período de amamentação do filho [20].

Ao analisar a renda familiar foi encontrada uma diferença significativa entre as doadoras $(R \$ 6.000,00$ para $D E$ e $R \$ 2.000,00$ para $D I, p<0,01)$ com valores distribuídos para aproximadamente 4 pessoas por família. Essa diferença é um resultado já esperado em virtude do perfil de atendimento do Instituto. De forma geral, as mulheres atendidas (DI) são de baixa renda e não possuem convênio de saúde, representando essa diferença.

Em contrapartida observa-se que em um universo com pessoas de baixa renda, a exemplo de países como os do continente africano, asiático e alguns latino-americanos, os recém-nascidos são nutridos com produtos industrializados. Em consequência há um risco moderado para desnutrição, infecção e crescimento nos índices de morbidade e mortalidade infantis [9].

Sabe-se que uma renda familiar elevada sugere um investimento em educação, o que consequentemente implicará na retenção e criticidade das informações passadas, bem como uma conduta proativa e responsável do indivíduo frente ao processo saúde-doença. Mulheres com essas características optam por gestar na fase adulta, com melhores oportunidades financeiras proporcionadas pela estabilidade profissional e com o apoio da sua rede social em especial o seu companheiro, justificando talvez os percentuais de manutenção de aleitamento materno exclusivo, representado pelas doadoras externas.

Ao analisar a tabela II, observa-se que a maioria das doadoras era primípara, resultado também encontrado no estudo de Alencar et. al. [1]. Essa condição pode ser um fator que leva as mulheres à procura de auxílio e de atendimento nos serviços de saúde, como reflexo de sua inexperiência e complicações do processo de amamentação. Essa procura por profissionais de saúde possibilita o acesso a informações acerca da existência dos BLH e a realização de doações [21].

Há uma tendência na redução das doações de LH à medida que aumenta o número de filhos. Uma realidade atribuída à redução de tempo livre das mães para ordenhar, preparar materiais e armazenar de forma correta o leite [22].

Observou-se também que as DE apresentaram um maior número de gestações, partos, filhos e abortamentos em comparação com as DI. Acreditamos que este resultado pode estar relacionado a uma média de idade superior das $D E$, vivenciando com maior frequência essas experiências obstétricas.

Ao analisar a história clínica, a doença que mais se destacou foi hipertensão arterial sistêmica (HAS), seguida por tuberculose. Destaca-se ainda que todas as doenças crônicas e infecciosas foram relatadas em sua maioria pelas doadoras internas do BLH. A maternidade em questão é uma unidade de saúde pública que atende a população carente às mais susceptíveis a comorbidades sugerindo, dessa forma, uma estreita relação entre a condição socioeconômica com o processo saúde-doença. Entretanto, acredita-se que a promoção e proteção à saúde são de corresponsabilidade da comunidade, do governo e da equipe multidisciplinar em saúde, seja no âmbito público ou privado [23]. 
Quando questionadas sobre hábitos de vida, somente 6,5\% $(n=2)$ DI referiram utilizar bebida alcoólica. Seu consumo faz parte do cotidiano de muitas mulheres, em especial aquelas com baixo nível socioeconômico, que por desconhecimento dos possíveis agravos ou por consequência do vício, mantém o uso durante a gravidez e a lactação. Devido aos fatores físicos e psíquicos envolvidos nos períodos de gravidez e amamentação, esses momentos tornam-se ideais para que os profissionais de saúde orientem a interrupção do uso de álcool e tabaco [24].

Quando questionadas sobre aspectos relacionados às mamas, a maioria delas referiu que estava em condições normais. Entretanto, quando comparamos os grupos, observou-se que grande parte das $\mathrm{DE}$, referiram mamas ingurgitadas. A dificuldade de amamentação, pega inadequada, ingurgitamento mamário, dentre outros, é o principal fator para a busca ao serviço de BLH, nesse momento o profissional deve orientar e sensibilizar a lactante e assim captar novas doadoras [22].

Esse resultado pode estar relacionado à assistência prestada pela equipe multiprofissional de saúde na maternidade escola. Por ser também um hospital amigo da criança, a instituição capacita e treina periodicamente seus funcionários para o manejo adequado das intercorrências resultando em menores números de complicações mamárias.

Entre os grupos de doadoras, ambos fizeram referência ao parto normal, representando 58,1\%. De acordo com Genovez et al. [25], o tipo de parto parece exercer influência no tempo de duração da amamentação e no risco de desmame precoce, assim como encontrado em outros estudos [26,27].

Quanto à realização das consultas de pré-natal, 93,5\% das doadoras de ambos os grupos realizaram a consulta de pré-natal, resultado que corrobora outros estudos $[1,24]$. $A$ consulta pré-natal faz-se de fundamental importância, pois é também, nesse momento, que a mãe receberá informações sobre aleitamento materno, preparação adequada para as mamas e realização de sorologias, sendo seus resultados úteis para o preenchimento dos pré-requisitos para a doação de LH.

Com relação às variáveis do nascimento do neonato, a maioria destes era a termo e tinha peso adequado para idade gestacional, resultado que corrobora estudo também desenvolvido em uma Maternidade de Referência de Salvador, que observou que $91,2 \%$ das doadoras tiveram seus bebês a termo (37 a 41 semanas) e 70,6\% nasceram com peso adequado [26]. Acreditamos que esse perfil de doadoras possa relacionar-se à procura do serviço do Instituto para o manejo de complicações mamárias, sendo dessa forma manejadas e posteriormente estimuladas a doarem o leite excedente. Com relação às $\mathrm{DI}$, essas mulheres provavelmente foram orientadas sobre a importância da doação ao BLH em virtude da oferta desse serviço na instituição.

Observa-se ainda uma diferença em relação ao tipo de aleitamento entre as doadoras, havendo um predomínio maior do aleitamento exclusivo, $86,7 \%$ entre as doadoras externas. Essa condição possivelmente está atrelada ao nível socioeconômico e escolaridade, com consequente disponibilidade de informações e conhecimentos sobre os benefícios da prática para o binômio, como encontrado em diversos estudos $[5,19,26]$. Entretanto as DI, apesar de terem o apoio do BLH da instituição, representaram um maior número de aleitamento misto, podendo estar relacionado a múltiplos fatores como: baixa qualidade do pré-natal e consequente ausência de orientação sobre aleitamento, falta de apoio à nutriz e baixa produção de leite em virtude da má alimentação e hidratação, condições intimamente relacionadas ao baixo nível socioeconômico.

É dado que para o sucesso do AM nos BLH faz-se necessário um padrão de normas a serem seguidas por todos os funcionários, treinamento continuo, fazer educação em saúde, incentivar e sugerir alternativas para a manutenção do AM, esclarecer sobre as desvantagens de uso de bico e mamadeiras, praticar o alojamento conjunto e direcionar as mães, com alta hospitalar, para grupos de apoio ao aleitamento materno na comunidade ou em serviços de saúde [28]. A última variável da pesquisa contempla as orientações dos profissionais de saúde sobre o aleitamento materno aos pais e familiares durante o pré-natal e mostrou que em comparação com as $\mathrm{DE}$, as DI tiveram em maior parte orientações relacionadas à prática durante esse período. Percebe-se dessa forma que os profissionais de saúde exercem grande influência no preparo da gestante durante a fase da amamentação, fato que nos leva a refletir sobre a responsabilidade das instituições em capacitação profissional e elaboração de estratégias para estímulo e apoio ao AM [29].

A educação em saúde bem como todo processo de preparo para lactação durante o pré-natal e pós-parto, comprovadamente, garante bons resultados para o AM e para 0 
crescimento das doações ao BLH. O profissional de saúde que mantém o contato com a gestante ou puérpera deve informar as vantagens da amamentação para o binômio mãe-filho, as desvantagens e técnicas apropriadas de amamentação a fim de prevenir e tratar ingurgitamento mamário, mastite, dor mamilar, abscesso mamário.

Além do domínio técnico os facilitadores do processo de amamentação que atuam no BLH necessitam ter carisma e empatia para promover a saúde emocional da mulher e realizar um atendimento humanizado [29].

Conclusão

O presente estudo permitiu identificar que as doadoras do BLH são mulheres na sua maioria casadas, primíparas, que tiveram acompanhamento de pré-natal, parto normal e mantiveram o aleitamento exclusivo. São assalariadas e possuem bom nível de escolaridade, corroborando o entendimento quanto à necessidade do aleitamento materno e sugere que 0 trabalho de incentivo a doação seja eficaz.

Ao identificar o perfil das doadoras do local de estudo, institui-se a possibilidade de futuros estudos e intervenções para captação de novas doadoras e fidelização das mesmas. Destacamos que o estudo realizado apresentou limitações quanto à sua população e amostra. $\mathrm{O}$ número de doadoras cadastradas no $\mathrm{BLH}$ da maternidade é superior ao número das participantes da pesquisa e a definição da amostra também é considerada um fator limitante, por não ser aleatória.

Logo, faz-se necessário investir em novas pesquisas e produções científicas específicas na área de Aleitamento Materno e Banco de Leite Humano, além de intensificar as orientações à genitora e sua família, núcleo social durante o pré-natal e puerpério, expandir a divulgação das ações dos $\mathrm{BLH}$, realizar a busca ativa de doadoras e capacitar profissionais de saúde para que sejam precursores da prática do aleitamento materno.

\section{Referências}

1. Alencar LCE, Seidl EMF, Fleury EM. Doação de leite humano: experiência de mulheres doadoras. Rev Saúde Pública 2009;43(1):70-7. https://doi.org/10.1590/s003489102009000100009

2. Pereira GC, Cardoso MVLML, Silva GRF, Bezerra MGA. Como e por que ser doadora de leite humano? Online Brazilian Journal of Nursing 2008;7(3):1-8.

3. Lamounier JA, Lana APB. Aleitamento materno. In: Novantino AF. Perinatologia Básica. 3 ed. Rio de Janeiro: Guanabara Koogan; 2006. p. 231-45.

4. Alves VH, Rodrigues DP, Branco MBLR, Souza RMP, Souza RRB, Medeiros FVA. Banco de leite humano na perspectiva da mulher doadora. Rev Rene 2013;14(6):116876.

5. Santos DT, Vannuchi MTO, Oliveira MMB, Dalmas JC. Perfil das doadoras de leite do banco de leite humano de um hospital universitário. Acta Scientiarum 2009;31(1):15-21.

6. Oliveira JSM. Percepção das doadoras de leite humano sobre doação e amamentação e suas características socioeconômicas e demográficas [Tese]. São Paulo: Faculdade de Saúde Pública (USP); 2010.

7. Alencar LCE, Seidl EMF. Doação de leite humano e apoio social: relatos de mulheres doadoras. Rev Latinoam Enferm 2010;18(3):381-9.

8. ONU. Nações Unidas do Brasil. Objetivos do Milênio até 2030. [citado 2017 Fev 19]. Disponível em: https://nacoesunidas.org/pos2015/ods3/

9. Pittas TM, Dri CF. O diálogo entre saúde e política externa na cooperação brasileira em bancos de leite humano. Ciênc Saúde Coletiva 2017;22(7);2277-86. https://doi.org/10.1590/1413-81232017227.02832017

10. Instituto de Perinatologia da Bahia [homepage na internet]. Banco de Leite Humano do IPERBA é credenciado em categoria ouro [citado 2016 Out 28]. Disponível em: http://saude.ba.gov.br/iperba/index.php?option=comcontent\&view=article\&id=49 .

11. Haddad N, Silva MB. Mortalidade feminina em idade reprodutiva no Estado de São Paulo, Brasil, 1991-1995: causas básicas de óbito e mortalidade materna. Rev Saúde Pública 2000;34(1):64-70. https://doi.org/10.1590/s0034-89102000000100012

12. Prado MM. Banco de Leite Humano do Município Pio de Varginha-MG: perfil das doadoras e causas de descarte de leite [Dissertação]. Lavras: Universidade Federal de Lavras; 2009. 
13. Machado RS, Campos CCP, Montoya JR, Schmidt RVJ. Experiencias de donación de leche humana en Andalucía-España: un estudio cualitativo. Enferm Glob 2015;14(1): 114-24.

14. Abreu J, Pereira Y, Lobato J, Foutoura I, Neto M, Santos F. Doação de leite materno: fatores que contribuem para esta prática. Arq Ciênc Saúde 2017;24(2):14-8. https://doi.org/10.17696/2318-3691.24.2.2017.548

15. Jeneral RB, Bellini LA, Duarte CR, Duarte MF. Aleitamento materno: uma reflexão sobre o papel do pai. Revista da Faculdade de Ciências Médicas de Sorocaba 2015;17(3):140-7.

16. Fragoso $A P R$, Fortes RC. Fatores associados à prática do aleitamento materno entre nutrizes de um hospital público do Distrito Federal. J Health Sci Int 2011;29(2):114-8.

17. Cavalcanti SH, Caminha MFC, Figueiroa JN, Serva VMSBD, Cruz RSBLC, Lira PIC et al. Fatores associados à prática do aleitamento materno exclusivo por pelo menos seis meses no estado de Pernambuco. Rev Bras Epidemiol 2015;18(1):208-19. https://doi.org/10.1590/1980-5497201500010016

18. Nakano AMS, Mamede MV. A mulher e o direito de amamentar: as condições sociais para o exercício desta função. Reme Rev Min Enf 2000;4(1/2):22-7.

19. Portal da saúde. [homepage na internet]. Ministério da Saúde quer dobrar salas de apoio à amamentação nas empresas. Brasil: Agência Saúde; [atualizado em 2008 Ago 07]; [citado 2015 Nov 16]. Disponível em:

http://portalsaude.saude.gov.br/index.php/cidadao/principal/agencia-saude/18972ministerio-da-saude-quer-dobrar-salas-de-apoio-a-amamentacao-nas-empresas.

20. Machado MOF, Parreira BDM, Dias FA, Costa NS, Monteiro JCS, Sponholz FG. Caracterização de nutrizes doadoras de um banco de leite humano. Ciênc Cuid Saúde 2013;12(3):529-38. https://doi.org/10.4025/cienccuidsaude.v12i3.18192

21. Rechia F, Cherubim D, Paula C, Padoin S. Fatores que interferem na doação de leite humano: revisão integrativa. Cogitare Enferm 2016;21(3):01-11.

22. Carvalho MS, ZequimMA. Doenças infecto-contagiosas relacionadas as carências habitacionais na cidade de Londrina-Paraná - Brasil. Scripta Nova. Revista Electrónica de Geografía y Ciencias Sociales 2003;7(146):113.

23. Chaves RG, Lamounier JAC, Cibele $C$. Fatores associados com a duração do aleitamento materno. J Pediatr 2007;83(3):241-6. https://doi.org/10.1590/S002175572007000400009

24. Genovez CB, Uchimiura TT, Satana R, Nishida FS. Banco de leite humano: uma análise das diferenças entre doadoras adultas e adolescentes. Acta Scientiarum Health Science 2011;33(2):211-8. https://doi.org/10.4025/actascihealthsci.v33i2.8266

25. Machado ACL, Santos JAD, Trigueiros PQS. Perfil das doadoras de leite materno do banco de leite humano de uma maternidade federal da cidade de Salvador, Bahia. Rev Ped SOPERJ 2017;17(2):18-24.

26. Lourenço D, Bardini G, Cunha L. Perfil das doadoras do banco de leite humano do Hospital Nossa Senhora da Conceição, Tubarão/SC. Arq Catarin Med 2012;41(1):22-7.

27. Silva CM, Pellegrinelli ALR, Pereira SCL, Passos LR, Santos LC. Práticas educativas segundo os "Dez passos para o sucesso do aleitamento materno" em um Banco de Leite Humano. Ciênc Saúde Coletiva 2017;22(5):1661-71. https://doi.org/10.1590/141381232017225.14442015

28. Siqueira SMC, Santos APR, Santos GA. Ações desencadeadas pelo enfermeiro para promoção do aleitamento materno e prevenção do desmame precoce. Revista Brasileira de Saúde Funcional 2017;1(1):56-65.

29. Marinho TF, Alves VH, Branco M BLR, Rodrigues DP, Pereira RM, Marchiori GRS. Percepções valorativas de práticas em banco de leite humano. Cogitare Enferm 2017;22(01):1-8. https://doi.org/10.5380/ce.v22i1.48679 